\title{
IUFOST2006/1319 \\ Processing concepts for non-thermal modification of foods
}

\author{
D. Knorr \\ Berlin University of Technology, Department Food Biotechnology and Food Process Engineering, \\ Koenigin-Luise-Str. 22, 14195 Berlin, Germany \\ dietrich.knorr@tu-berlin.de
}

\begin{abstract}
Non-thermal processing concepts such as high hydrostatic pressure (HP), high intensity pulsed electric fields (PEF) or combinations with mild heat or antimcrobial agents have proven to be effective and gentle means of food preservation. Such processes can also be utilized to design processing concepts for specific notification of foods or food constituents. High pressure modification of proteins and polysaccharides can aid the development of new gel structures with selected physico-chemical properties. Thermoresistance of enzymes under moderate pressure conditions (approx. $250 \mathrm{MPa}$ ) can be increased up to $30^{\circ} \mathrm{C}$ thus enabling targeted bioconversations at elevated temperatures. The temperature resistance of probiotic strains could be increased by applying pressure treatment of $100 \mathrm{MPa}$ and subsequent production of stress proteins. Further, resistance of starches to digestion could be increased via pressure treatment. Stress induction via HP or PEF processing has been used for metabolite engineering in plant systems resulting in increased production of oils, phenols, phytosterols or glucosinolates. Future research needs and current challenges in the development of such processing concepts will also be addressed.
\end{abstract}

